\title{
Determination of plasma velocity from light fluctuations in a cutting torch
}

\author{
L. Prevosto, $\left.{ }^{1, a}\right)$ H. Kelly, ${ }^{2}$ and B. Mancinelli ${ }^{1}$ \\ ${ }^{1}$ Departamento Ing. Electromecánica, Grupo de Descargas Eléctricas, Universidad Tecnológica Nacional, \\ Regional Venado Tuerto, Las Heras 644, Venado Tuerto 2600, Pcia. Santa Fe, Argentina \\ ${ }^{2}$ Departamento de Física, Instituto de Física del Plasma (CONICET), Facultad de Ciencias Exactas \\ y Naturales (UBA), Ciudad Universitaria Pab. I, 1428 Buenos Aires, Argentina
}

(Received 8 July 2009; accepted 5 August 2009; published online 11 September 2009)

\begin{abstract}
Measurements of plasma velocities in a $30 \mathrm{~A}$ high energy density cutting torch are reported. The velocity diagnostic is based on the analysis of the light fluctuations emitted by the arc which are assumed to propagate with the flow velocity. These light fluctuations originate from plasma temperature and plasma density fluctuations mainly due to hydrodynamic instabilities. Fast photodiodes are employed as the light sensors. The arc core velocity was obtained from spectrally filtered light fluctuations measurements using a band-pass filter to detect light emission fluctuations emitted only from the arc axis. Maximum plasma jet velocities of $5000 \mathrm{~m} \mathrm{~s}^{-1}$ close to the nozzle exit and about $2000 \mathrm{~m} \mathrm{~s}^{-1}$ close to the anode were found. The obtained velocity values are in good agreement with those values predicted by a numerical code for a similar torch to that employed in this work. (C) 2009 American Institute of Physics. [doi:10.1063/1.3213377]
\end{abstract}

\section{INTRODUCTION}

Plasma cutting torches produce a hot, highly constricted, and high velocity arc plasma jet between a cathode and a work-piece acting as the anode. That plasma is created by a narrow nozzle inside the torch, where a gas is injected at a high pressure. ${ }^{1}$ In order to obtain high-quality square cuts of narrow kerfs without dross, it is necessary to transfer a sufficient amount of heat and momentum to the work-piece so this work-piece can be melted with the molten metal removed from the cut. Consequently, three parameters in a plasma cutting process become relevant: the plasma temperature $(T)$, the plasma density $(n)$, and the plasma velocity $(v)$. These parameters determine the enthalpy and the momentum fluxes carried by the plasma flow, so their knowledge allows the process improvement and also the validation of theoretical arc models. Many experimental investigations related to the determination of the plasma temperature and density exists in the literature, but there are no reports on the determination of the cutting arc velocity. Measurements of $T$ and $n$ have been reported in the literature, generally from nonintrusive techniques as emission spectroscopy ${ }^{2-4}$ and more recently using Langmuir probe techniques. ${ }^{5-7}$ The lack of measurements of plasma velocities for cutting torches is probably due to the difficulties in implementing conventional fluid diagnostics (laser Doppler anemometry, laser light scattering, Doppler line shift, enthalpy probes, etc.) to determine very high plasma velocities in an extremely high-enthalpy, high density plasma. In particular, published numerical codes for cutting $\operatorname{arcs}^{3-8}$ indicate that the plasma flow velocity can reach very high values, of the order of $5 \times 10^{3} \mathrm{~m} / \mathrm{s}$ at the arc core for a 30 A cutting torch and $8 \times 10^{3} \mathrm{~m} / \mathrm{s}$ for a $200 \mathrm{~A}$ arc.

On the other hand, there are some reports on the determination of $v$ in high-pressure noncutting thermal arcs, using

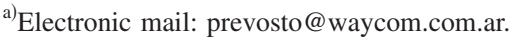

optical techniques based on the analysis of the fluctuations of the light emitted by the $\operatorname{arc}^{9-11}$ and intrusive techniques as enthalpy probe methods. ${ }^{12}$

In this work, measurements of plasma jet velocity in a 30 A high energy density cutting torch are reported. The method is based on the analysis of the light fluctuations emitted by the arc which are assumed to propagate with the flow velocity. Fast photodiodes are employed as the light sensors. The plasma motion is detected either by the cross-correlation of fluctuating signals, ${ }^{10,11}$ which are simultaneously measured at different axial positions along the arc, or by the application of the fast Fourier transform (FFT) algorithms ${ }^{9}$ to the such fluctuating data.

The organization of the paper is as follows. In Sec. II the experimental arrangement is presented. The measured values for the plasma jet velocity are presented and discussed in Sec. III and the conclusions are drawn in Sec. IV.

\section{EXPERIMENTAL ARRANGEMENT}

The schematic of the experimental setup is shown in Fig. 1. The low current (30 A) high energy density cutting torch used in this study consisted of a cathode centered above an

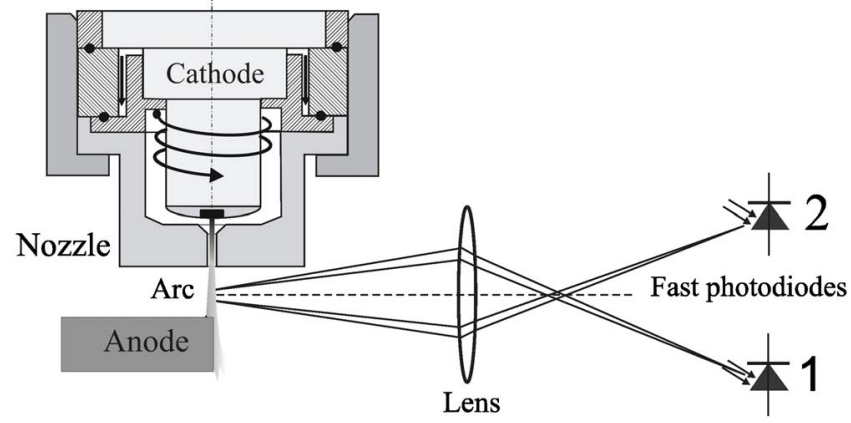

FIG. 1. (Color online) Schematic of the arc torch and the light detection system. 
orifice in a copper nozzle without liquid cooling. The cathode was made of copper ( $7 \mathrm{~mm}$ in diameter) with a hafnium tip (1.5 $\mathrm{mm}$ in diameter) inserted at the cathode center. A flow of oxygen gas cooled the cathode and the nozzle and was also employed as the plasma gas. The gas passed through a swirl ring to provide arc stability. The nozzle consisted in a converging-straight bore (with a bore radius of 0.5 $\mathrm{mm}$ and a length of $4.5 \mathrm{~mm}$ ) in a copper holder surrounding the cathode. To avoid plasma contamination by metal vapors from the anode, a rotating steel disk (rotating frequency of the disk equal to $23 \mathrm{~s}^{-1}$ ) with $200 \mathrm{~mm}$ diameter and $11 \mathrm{~mm}$ thickness was used as the anode. ${ }^{3,5}$ The disk's upper surface was located at $6 \mathrm{~mm}$ from the nozzle exit. A well-stabilized arc column was obtained, and no damages on the lateral surface of the anode disk could be detected. Thus, practically no metal vapors from the anode were present in the arc. More details on the torch characteristics can be found elsewhere. ${ }^{13,14}$ The optical system consisted simply in a converging lens (with $50 \mathrm{~mm}$ diameter and $100 \mathrm{~mm}$ focal distance) located at $114 \mathrm{~mm}$ from the arc axis that produced a magnified $(7 \times)$ image of the arc on a plane located at 914 $\mathrm{mm}$ from the arc axis. On that image plane were mounted two fast photodiodes (BPX 65) on a grounded copper holder and separated $7 \mathrm{~mm}$ in the axial direction. Since the sensitive area of the photodiodes was $1 \times 1 \mathrm{~mm}^{2}$, these sensors were collecting light from two $0.14 \times 0.14 \mathrm{~mm}^{2}$ arc regions separated by $1 \mathrm{~mm}$ in the axial direction. Due to its large magnification, the optical system had a very small depth of field, meaning that in practice the light collected by each photodiode came from a distance of $\pm 0.5 \mathrm{~mm}$ in a direction perpendicular to the arc axis (measured from the arc center). This spatial resolution in the radial direction was very close to the arc diameter (see below). The photodiodes were negatively biased to $-9 \mathrm{~V}$, and their photocurrents were registered as voltage signals on a $12 \mathrm{k} \Omega$ resistance. These signals were registered in a two-channel digitizing oscilloscope Tektronix TDS 1002 B with a sampling rate of $500 \mathrm{MS} / \mathrm{s}$ and an analogical bandwidth of $60 \mathrm{MHz}$. The frequency response of the detecting circuit was limited by that resistance and the stray parallel capacity of the measuring coaxial cable plus the oscilloscope input capacity, resulting in a cutoff upper frequency of $5 \mathrm{MHz}$. In order to reduce the electromagnetic noise in the photodiode signals, it was necessary to build the photodiodes holder with thick copper walls $(4 \mathrm{~mm})$. In this condition the noise amplitude could be reduced to less than 1 $\mathrm{mV}$, while typical light amplitudes varied in the range 2-50 $\mathrm{mV}$.

\section{EXPERIMENTAL RESULTS AND DISCUSION}

As quoted in Sec. I, a cross-correlation technique was used. Only frequencies $(f)$ within the range $1 \mathrm{kHz} \leq f$ $\leq 1 \mathrm{MHz}$ were considered, so we applied a FFT band-pass filter to the acquired signals. The lower frequency limit was selected to avoid fluctuations induced by the ripple of the arc power source (with a fundamental frequency of $50 \mathrm{~Hz}$ and several high-order harmonics), while the upper limit was chosen in according to the cutoff frequency of the detecting circuit. A FFT was applied to each pair of photodiode sig- nals. As it is well known, the FFT gives an array of complex numbers that contain information on the amplitudes and phases corresponding to each elementary harmonic into which the recorded signal is decomposed. If the fluctuations are carried by the flow, the phase difference $(\Delta \Phi)$ between the two photodiode signals should be a linear function of the frequency, and the flow velocity can be derived from the slope of this linear function as ${ }^{9}$

$$
v=2 \pi d \frac{\Delta f}{\Delta \Phi},
$$

where $d$ is the distance between the photodiodes (in this case, $d=1 \mathrm{~mm}$ ). Alternatively, the delay $(\tau)$ between both signals can be calculated from the maximum of the cross-correlation in the time domain, and hence the flow velocity is simply calculated as

$$
v=\frac{d}{\tau} .
$$

One of the main problems in applying the quoted technique to cutting torches is the very thin radial dimension of the arc (on the order of the radial resolution of the measurement), together with the large radial velocity gradients predicted by the numerical codes. ${ }^{3,4,8}$ Both characteristics make it quite difficult to identify the arc region that is being focused, and which one is the velocity associated to that focused region. However, the relatively simple shape of the emitted spectra by an oxygen torch allows clearing the quoted difficulty. The complete visible spectrum (400-850 nm) of a low current cutting torch operated with oxygen gas and using a rotating disk as the anode was published by Freton et al. ${ }^{3}$ One can see from that work that the spectrum is composed by a series of lines superimposed on a continuum emission. According to the National Institute of Standards and Technology spectral tables, ${ }^{15}$ all the lines appearing in the spectral window 400$500 \mathrm{~nm}$ (see Fig. 8 of the quoted work) correspond to ionized atomic oxygen $\left(\mathrm{O}^{+}\right)$, while the lines in the window 600-850 $\mathrm{nm}$ correspond to atomic oxygen $(\mathrm{O})$. No molecular emission was identified in this spectrum. On the other hand, for the typical range of $T$ values measured in cutting torches $\left[(12-20) \times 10^{3} \mathrm{~K}\right]$, continuum emission is dominated by recombination radiation (free bound). ${ }^{16}$ The recombination radiation is proportional to the electron and ion densities (i.e., proportional to the square of the plasma density). Since the excitation processes in thermal plasmas are mainly due to electron impact, the line radiation from ionized oxygen is also proportional to the square of the plasma density, while line radiation from atomic oxygen is proportional to the neutral oxygen and plasma densities. Besides, the plasma emission coefficients strongly increase with $T$, within the typical range of $T$ values found in the arc. In Fig. 2 the radial profiles of $T$, and the ionized $\left(n_{\mathrm{O}}{ }^{+}\right)$and atomic oxygen $\left(n_{\mathrm{O}}\right)$ densities are, respectively, shown. The $T$ profile was taken from Ref. 6 and corresponds to an axial position located at $3.5 \mathrm{~mm}$ from the nozzle exit, and at an arc pressure of 0.1 $\mathrm{MPa}$. The densities were calculated from the $T$ profile assuming local thermodynamic equilibrium and using the equation of state. As it can be seen, $n_{\mathrm{O}}{ }^{+}$is negligible for 


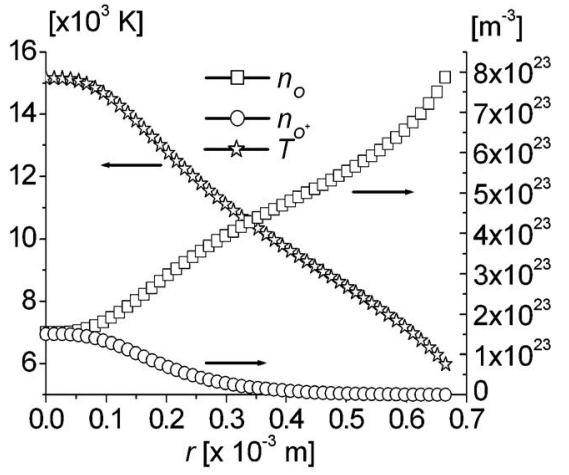

FIG. 2. Radial profiles of the plasma temperature $(T)$, and the ionized $\left(n_{\mathrm{O}}{ }^{+}\right)$ and atomic oxygen $\left(n_{\mathrm{O}}\right)$ densities at $z=3.5 \mathrm{~mm}$ from the nozzle exit.

radial distances larger than $0.35 \mathrm{~mm}$. On the other hand, $n_{\mathrm{O}}$ increases continuously from the arc center (where the ionization degree is about $50 \%$ ) to the arc periphery. So in practice it can be assumed that the $\mathrm{O}^{+}$emission comes from a hot and dense thin zone $(\sim 0.1 \mathrm{~mm})$ around the arc axis, whereas the $\mathrm{O}$ emission comes from the arc edge where there is a considerable number of atomic oxygen. The continuum radiation is also emitted mainly from the arc center.

Following the described arc emissive structure, it was decided to filter the arc light using a band-pass filter centered in $475 \mathrm{~nm}$ and with a $50 \mathrm{~nm}$ bandwidth. In Fig. 3 typical photodiode signals registered in this condition are presented. The smoothed signals (obtained after applying the quoted FFT band-pass filter) are also shown. In this figure, one of the photodiode (number 1) was focused at $0.5 \mathrm{~mm}$ from the nozzle exit, while the other (number 2) at $1.5 \mathrm{~mm}$ from the nozzle exit. So the derived flow velocity was ascribed to an averaged axial position of $1 \mathrm{~mm}$ from the nozzle exit. In Fig. 4(a), the phase difference between the two photodiode signals as a function of the frequency is shown. It can be seen that an approximate linear behavior between $\Delta \Phi$ and $f$ is observed for $f<800 \mathrm{kHz}$, giving evidence that the light fluctuations in the quoted $f$ range are effectively carried by the flow. Equation (1) predicts then a flow velocity of $5 \pm 1 \times 10^{3} \mathrm{~m} / \mathrm{s}$. In Fig. 4(b), the cross-correlation in the time domain is plotted. From this figure, one obtains $\tau$ $=0.2 \pm 0.01 \times 10^{-6} \mathrm{~s}$ and Eq. (2) gives $v=5 \pm 0.3$ $\times 10^{3} \mathrm{~m} / \mathrm{s}$. The uncertainty in the $\tau$ value is mainly of sta-

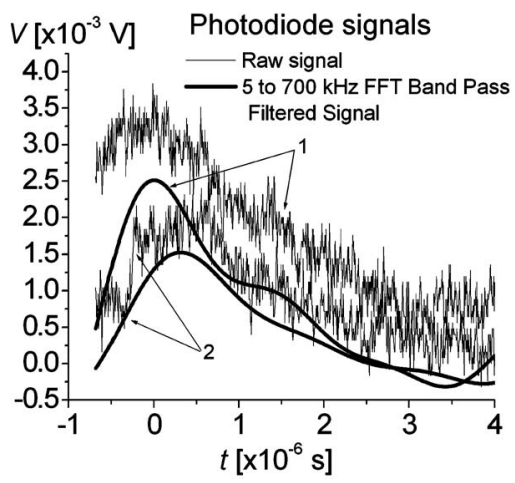

FIG. 3. Typical photodiode signals. The signal labeled 1 corresponds to the photodiode focusing at $z=0.5 \mathrm{~mm}$ from the nozzle exit. The signal 2 corresponds to the photodiode focusing at $z=1.5 \mathrm{~mm}$.
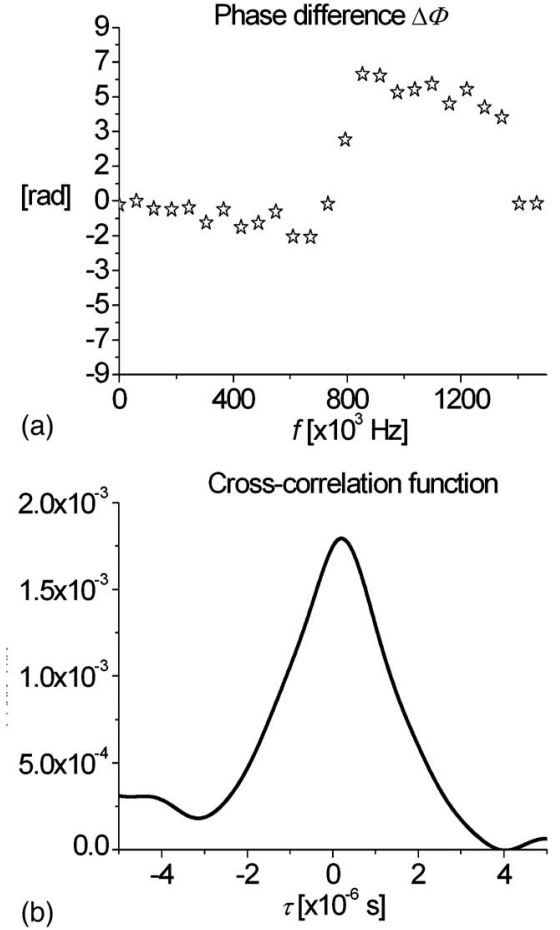

FIG. 4. (a) Phase difference $(\Delta \Phi)$ between the two photodiode signals as a function of the frequency $(f)$. (b) Cross-correlation function in the time domain.

tistical origin. Although both derived velocity values were coincident within the experimental uncertainty, the crosscorrelation method appeared to be more appropriated in this case because the sampling time interval was not too small as compared with the $\tau$ value (thus giving a relatively small number of points in the phase plot).

In Fig. 5, the flow velocity obtained for different axial positions ( $z$, measured from the nozzle exit) are presented. Each point in the graph represents an average of several different measurements performed under similar conditions. A visible photograph of the arc image (showing a complex light pattern from the nozzle exit up to $z \sim 2 \mathrm{~mm}$ ) is inserted in the figure. According to a published numerical code, ${ }^{3}$ this pattern is due to a fast expansion of the flow from the nozzle exit (with an associated pressure drop down to subatmospheric pressure values), which is followed by a steady shock

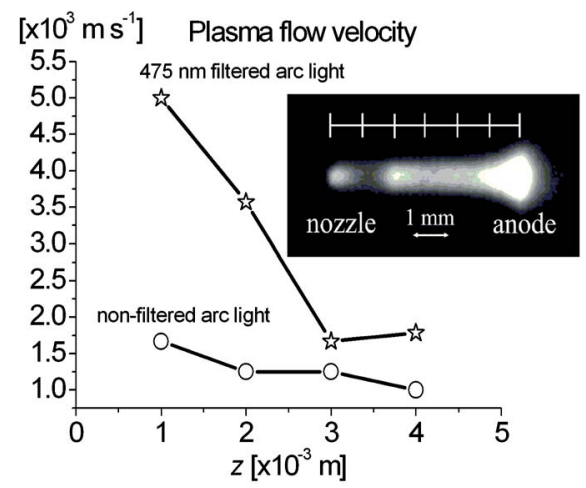

FIG. 5. (Color online) Flow velocity $(v)$ obtained for different axial positions $(z)$ with and without band-pass filter. A visible photograph of the arc is shown in the inset $(z=0$ coincides with the nozzle exit). 
wave that matches the arc pressure to the atmospheric value. During this phenomenon a turbulent mixing (due to the development of hydrodynamic instabilities of the KelvinHelmholtz type $\mathrm{e}^{17,18}$ ) with the surrounding cold oxygen gas at the arc periphery could become relevant and gives place to the transport of successive plasma volumes with different densities and temperatures that produce the light fluctuations. It can be seen that the obtained velocity strongly decreases with $z$. For $z=1 \mathrm{~mm}$, the flow velocity is supersonic, while at $z=2 \mathrm{~mm}$ the flow becomes subsonic, in correspondence with the presence of a shock. The obtained velocity values are very close to those obtained at the arc axis by a numerical $\operatorname{code}^{3}$ that includes turbulence effects, predicting a strong flow deceleration along the $z$ coordinate from $z=1 \mathrm{~mm}$. For comparison purposes, flow velocities obtained without light filtering are also plotted in Fig. 5. It can be seen that the flow velocities obtained from the nonfiltered light are much smaller than those previously obtained with the filter. In this condition, the photodiodes are detecting a large number of red lines which are presumably excited at the arc border. It is worth noting that the photodiodes are much more sensitive to red light, and in practice the photodiode signals increased by about a factor of 20 when nonfiltered light was detected.

\section{CONCLUSIONS}

We have presented measurements of plasma jet velocity in a 30 A high energy density cutting torch based on the detection of light fluctuations that are carried by the flow. Although this method have been employed successfully in nontransferred arc torches, to our knowledge it is the first time their use is reported in cutting torches, allowing the determination of very high plasma velocities, of the order of thousands of meters per second. The obtained velocity values are in good agreement with those values predicted by a numerical code for a torch similar to that employed in this work.

\section{ACKNOWLEDGMENTS}

This work was supported by grants from the Universidad de Buenos Aires (Grant No. PID X111), CONICET (Grant No. PIP 5378), and UTN (Grant No. PID Z 012). One of the authors (L.P.) is grateful to the Fundación YPF. Also B.M. is grateful to the Universidad Tecnológica Nacional. H.K. is a member of the CONICET.

${ }^{1}$ V. A. Nemchinsky and W. S. Severance, J. Phys. D 39, R423 (2006)

${ }^{2}$ L. Girard, Ph. Teulet, M. Razafinimanana, A. Gleizes, F. Camy-Peyret, E. Baillot, and F. Richard, J. Phys. D 39, 1543 (2006).

${ }^{3}$ P. Freton, J. J. Gonzalez, A. Gleizes, F. Camy Peyret, G. Caillibotte, and M. Delzenne, J. Phys. D 35, 115 (2002).

${ }^{4}$ P. Freton, J. J. Gonzalez, F. Camy Peyret, and A. Gleizes, J. Phys. D: Appl. Phys. 36, 1269 (2002).

${ }^{5}$ L. Prevosto, H. Kelly, and B. Mancinelli, IEEE Trans. Plasma Sci. 36, 263 (2008).

${ }^{6}$ L. Prevosto, H. Kelly, and B. Mancinelli, IEEE Trans. Plasma Sci. 36, 271 (2008)

${ }^{7}$ L. Prevosto, H. Kelly, and B. Mancinelli, IEEE Trans. Plasma Sci. 37, 1092 (2009).

${ }^{8}$ Q. Zhou, H. Li, X. Xu, F. Liu, S. Guo, X. Chang, W. Guo, and P. Xu, J. Phys. D 42, 015210 (2009).

${ }^{9}$ N. Singh, M. Razafinimanana, and J. Hlinas, J. Phys. D 33, 275 (2000).

${ }^{10}$ M. P. Planche, J. F. Coudert, and P. Fauchais, Plasma Chem. Plasma Process. 18, 263 (1998).

${ }^{11}$ W. Hermann, U. Kogelschatz, L. Niemeyer, K. Regaller, and E. Schade, J. Phys. D 7, 1703 (1974).

${ }^{12}$ F. Xiaozhen, Plasma Sci. Technol. 5, 1909 (2003).

${ }^{13}$ L. Prevosto, H. Kelly, and B. Mancinelli, J. Appl. Phys. 105, 013309 (2009).

${ }^{14}$ L. Prevosto, H. Kelly, and B. Mancinelli, J. Appl. Phys. 105, 123303 (2009).

${ }^{15}$ NIST Atomic Spectra Database. Available at http://physics.nist.gov/ PhysRefData/ASD/lines.

${ }^{16}$ Y. B. Zel'dovich and Y. P. Raizer, Physics of Shock Waves and HighTemperature Hydrodynamic Phenomena (Academic, New York, 1966), Vol. 1.

${ }^{17}$ G. R. Jones and M. T. C. Fang, J. Phys. D 43, 1415 (1980).

${ }^{18}$ J. D. Yan, K. I. Nuttall, and M. T. C. Fang, J. Phys. D 32, 1401 (1999). 